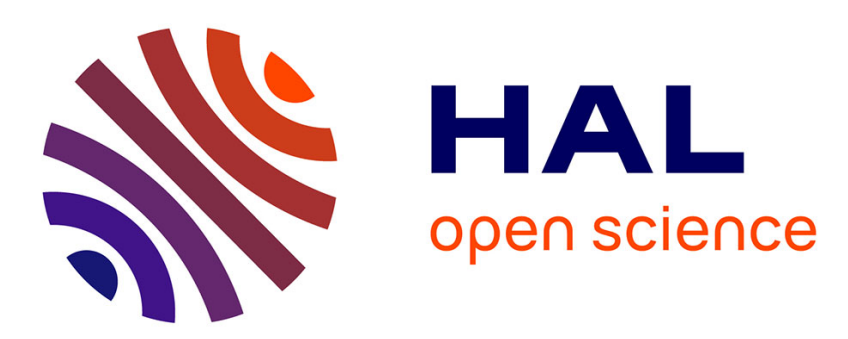

\title{
The SUMO protease SENP7 is a critical component to ensure HP1 enrichment at pericentric heterochromatin.
}

Christèle Maison, Kelly Romeo, Delphine Bailly, Marion Dubarry, Jean-Pierre Quivy, Geneviève Almouzni

\section{- To cite this version:}

Christèle Maison, Kelly Romeo, Delphine Bailly, Marion Dubarry, Jean-Pierre Quivy, et al.. The SUMO protease SENP7 is a critical component to ensure HP1 enrichment at pericentric heterochromatin.. Nature Structural and Molecular Biology, 2012, 19 (4), pp.458-60. 10.1038/nsmb.2244 . hal-00765968

\section{HAL Id: hal-00765968 \\ https://hal.science/hal-00765968}

Submitted on 17 Dec 2012

HAL is a multi-disciplinary open access archive for the deposit and dissemination of scientific research documents, whether they are published or not. The documents may come from teaching and research institutions in France or abroad, or from public or private research centers.
L'archive ouverte pluridisciplinaire HAL, est destinée au dépôt et à la diffusion de documents scientifiques de niveau recherche, publiés ou non, émanant des établissements d'enseignement et de recherche français ou étrangers, des laboratoires publics ou privés. 
The SUMO protease SENP7 is a critical component to ensure HP1 enrichment at pericentric heterochromatin

Christèle Maison*, Kelly Romeo*, Delphine Bailly, Marion Dubarry, Jean-Pierre Quivy ${ }^{\ddagger}$ and Geneviève Almouzni ${ }^{\ddagger}$

Institut Curie, Centre de recherche, Paris, France

Centre National de la Recherche Scientifique (CNRS), Unité Mixte de Recherche UMR218, Laboratory of Nuclear Dynamics and Genome Plasticity, Paris, France

\footnotetext{
* These authors contributed equally to this work

${ }^{\ddagger}$ Corresponding authors
}

For correspondence: G. Almouzni

Tel: + $33(0) 156246701$

Fax: + $33(0) 146333016$

E-mail: geneviève.almouzni@curie.fr 
SUMOylation promotes targeting of HP1a to pericentric heterochromatin. Here we identify the SUMO protease SENP7 as a maintenance factor for HP1 $\alpha$ accumulation at pericentric heterochromatin. SENP7 interacts directly with HP1a, localizes at HP1enriched pericentric domains and can deconjugate sumoylated HP1a in vivo. Depletion of SENP7 delocalizes HP1 $\alpha$ from pericentric heterochromatin without affecting H3K9me3. We propose that following targeting of HP1a, a subsequent desumoylation event enables HP1a retention at these domains.

HP1, a protein highly conserved throughout evolution ${ }^{1,2}$, interacts with many partners $^{2,3}$ that contribute to its enrichment at pericentric heterochromatin ${ }^{4}$. Our recent discovery that HP1a sumoylation promotes its targeting to pericentric heterochromatin ${ }^{5}$ in mouse cells raised the possibility that following this step, desumoylation could favour HP1a maintenance at these domains. Sumoylation is rapidly reversed by the action of SUMOspecific proteases, known as SENPs ${ }^{6-10}$. There are six SENPs in mammals (SENP1-3, SENP5-7 $)^{8,9}$, whose exact targets are not defined ${ }^{7,9}$. The presence of SENP7 in the recent list of 82 candidate interactors of the human HP1 $\alpha^{3}$ prompted us to further explore a role for this SENP as a SUMO regulator impacting HP1 stability at pericentric heterochromatin.

First, we verified that the association of SENP7 with HP1a was conserved in mouse. We found that both wild type GFP-tagged mouse SENP7 (e-SENP7) and catalytically-dead C979S $^{11}$ (e-SENP7M) coimmunoprecipitated with endogenous HP1a but not with KAP1 or SetDB1 (Fig. 1a and Supplementary Fig. 1a). We further confirmed that GST-SENP7 pulled down the three endogenous HP1 isoforms (Supplementary Fig. 1b). Conversely, HA-tagged HP1 1 (e-HP1 $)$ retrieved endogenous SENP7 but not the most closely related SENP, SENP6 (Fig. 1b), indicating that HP1a associates specifically with SENP7 regardless of its protease activity. Finally using recombinant proteins, we determined that His-SENP7 interacted directly with purified GST-HP1a (Fig. 1c). At a cellular level, endogenous SENP7 colocalizes with HP1 $\alpha$ at pericentric heterochromatin and remains there after extraction of soluble material with Triton as shown by immunofluorescence (Fig. 2a). In an asynchronous 
cell population, $70 \%$ of the cells showed a co-enrichment of HP1 $1 \alpha$ and SENP7 at the pericentric domain while the remaining $30 \%$ of cells showed diffuse staining for both proteins (Fig. 2b). We then investigated SENP7 localization at pericentric heterochromatin under conditions impairing localization of HP1 isoforms at these domains (after RNase A treatment on NIH3T3 cells ${ }^{12}$ or in histone methyltransferase Suv39h double-null MEFs ${ }^{12,13}$ ). Notably, both situations erased SENP7 staining at pericentric domains (Fig. 2c,d) without affecting the total amount of SENP7 and HP1 protein. These data demonstrate that the localization of SENP7 parallels that of HP1a.

Since HP1 $\alpha$ directly interacts with SENP7 and is modified by SUMO-1 in vivo ${ }^{5}$, we examined whether SENP7 could affect the levels of SUMO-1-HP1a in vivo. To obtain detectable SUMO-1-HP1 levels, we co-transfected GFP-SUMO-1 and HP1 - -HA into NIH3T3 cells. Under conditions preserving SUMO modification and after immunoprecipitation of HP1 1 -HA, we revealed a $70 \mathrm{kD}$ diagnostic band corresponding to GFP-SUMO-1-HP1 $\alpha-H A$ (Fig. 2e and Supplementary Figs. 2 and 3). Remarkably, this band decreased in intensity upon introduction of an active GFP-SENP7 (Fig. 2e and Supplementary Figs. 2 and 3) and remains identical to control when using the catalytic-dead mutant SENP7C979S ${ }^{11}$ expressed at levels comparable to GFP-SENP7 (Fig. 2e). Thus, under our experimental conditions, SENP7 can deconjugate SUMO-1-modified HP1a in vivo.

To further explore a functional relationship between the two proteins, we tested whether the SENP7-HP1 $\alpha$ interaction could impact HP1 $\alpha$ maintenance at pericentric domains. We depleted SENP7 from NIH3T3 cells by transfecting a plasmid encoding both a miRNA to downregulate SENP7 (miSENP7) and a GFP mRNA to enable identification of the transfected cells (Fig. 3a and see Supplementary Fig. 4a,b for depletion efficiency). Cell viability and FACS profiles based on DNA staining remained comparable between control and downregulated cells (Supplementary Fig. 4c). However, only $44 \%$ of GFP-positive SENP7-depleted cells showed HP1a localization at pericentric heterochromatin compared to $70 \%$ of GFP-positive control cells (Fig. 3b,c), indicating a significant decrease of HP1a localization at pericentric domains $\left(p_{\text {value }}=0.004\right)$. In these SENP7-depleted cells, HP1 $\alpha$ 
showed faint staining at pericentric domains and increased diffuse nuclear staining (compare top rows of miSENP7 and micont in Fig. 3b) while keeping a normal amount of HP1 protein and a dense DAPI staining corresponding to pericentric regions (Supplementary Fig. 4a,b). Remarkably, although SENP7 depletion leads to the loss of local HP1a enrichment at pericentric heterochromatin, $\mathrm{H} 3 \mathrm{~K} 9 \mathrm{me} 3$ remained present at these domains (Fig. 3d,e), as found when depleting Orc proteins ${ }^{14}$. We confirmed these results by siRNA depletion (Supplementary Fig. 4d-f). Next, to evaluate if this effect on HP1 $\alpha$ was specific to SENP7, we examined SENP6, which did not colocalize with HP1 $\alpha$ and SENP6 depletion did not alter HP1a localization (Fig. 3f,g and Supplementary Fig. 4g). However, we noted that SENP6 was able to deconjugate sumoylated HP1a in the transfection assay (Supplementary Fig. 5). Taken together, these data underline the fact that the importance of SENP7 in maintaining HP1 $\alpha$ enrichment at pericentric domains likely involves the specificity of SENP7 localization. The loss of SENP7 and HP1 without affecting the H3K9me3 mark nor the overall organization of the domains emphasizes the existence of distinct steps for HP1 accumulation at pericentric domains.

In conclusion, we propose that SENP7 is a novel maintenance factor for HP1a accumulation at pericentric heterochromatin based on the following arguments : (i) its direct interaction with HP1, (ii) the common behaviour and localization of the two proteins at pericentric heterochromatin and (iii) loss of HP1 $\alpha$ enrichment at pericentric heterochromatin after SENP7 depletion. Moreover, we identify SUMO-1-modified HP1a as a substrate for SENP7. Although sumoylation is a post-translational modification involved in many cellular processes, surprisingly, only a few putative substrates have been identified for the SENPs ${ }^{7,9}$, and to our knowledge, none have been identified for SENP7. While previous studies in vitro indicated a SENP7 deconjugating activity for SUMO2/3 $3^{15}$ and a limited activity for SUMO- $1^{11}$, we provide evidence that SENP7 could equally remove SUMO-1 or SUMO-2 in vivo using exogenously expressed SUMO (Supplementary Fig. 6). Thus, given that endogenous HP1a is modified by SUMO-1, but not SUMO-2/3, when associated with major RNA as recently detected $^{5}$, it is tempting to consider that SENP7 role in regulating HP1a accumulation at 
pericentric heterochromatin could serve to remove SUMO-1. We do not exclude however possible role to remove SUMO-2/3. To target the activity of SENP7 on SUMO-1-HP1a, the simplest options to consider are the direct interaction between SENP7 and HP1 $\alpha$ combined with the localization of SENP7 at HP1-enriched domains. However, we do not exclude the possibility that SENP7 may deconjugate other sumoylated factors present at pericentric domains, such as KAP1, a known HP1 interacting partner that is SUMO-1-modified in vivo ${ }^{16}$. Our data place the cycle of sumoylation/desumoylation as an important new player in regulating efficient accumulation of $\mathrm{HP} 1 \alpha$ at pericentric domains. After a SUMO-dependent targeting of HP1 $1 \alpha$ to pericentric heterochromatin ${ }^{5}$, a subsequent retention step would be provided by SENP7 desumoylation activity. Interestingly, an HP1 $\alpha$-SUMO-1 fusion protein that remains constitutively sumoylated can be rapidly and efficiently targeted to pericentric heterochromatin, yet it proved toxic to the cells ${ }^{5}$. This indicates that persistence of the SUMO modification on HP1 should be avoided. While SENP7 is likely to be the specific SUMO protease acting at these domains on modified HP1, future work should aim to identify the HP1 specific SUMO ligase machinery in order to decipher how this cycle can be regulated.

\section{ACKNOWLEDGEMENTS}

We thank Ron Hay for reagents, Claude Gazin for early investment, Sylvain Cantaloube for technical help, Alysia vandenBerg for critical reading of the manuscript, members of the unit for discussions and the Curie Imaging platform for microscopy facility. K. Romeo received support from University Pierre et Marie Curie (UPMC), Paris, France/Ministère de l'Enseignement Supérieur et de la Recherche. This work was supported by la Ligue Nationale contre le Cancer (Equipe labellisée Ligue 2010), the European Commission Network of Excellence EpiGeneSys (HEALTH-F4-2010-257082), ERC Advanced Grant 2009-AdG_20090506 "Eccentric" and ANR "ECenS" ANR-09-BLAN-0257-01. 


\section{AUTHOR CONTRIBUTIONS}

$\mathrm{JPQ}, \mathrm{CM}$ and $\mathrm{GA}$ conceived and designed the experiments. $\mathrm{KR}, \mathrm{CM}, \mathrm{DB}, \mathrm{MD}$ and JPQ performed the experiments. JPQ, CM, KR and GA analysed the data. JPQ, CM and GA wrote the paper.

\section{COMPETING FINANCIAL INTERESTS}

The authors declare no competing financial interests.

\section{FIGURE LEGENDS}

Figure 1 SENP7 associates with HP1a. (a) Immunoprecipitation (IP) of GFP (control), GFPSENP7 wild-type (e-SENP7) and GFP-SENP7C979S (e-SENP7M). Input (Inp) is 5, 2.5 and $1.25 \%$ of total cell extract. (b) Immunoprecipitation (IP) of hnRNPC-HA (e-hnRNPC) and HP1a-HA (e-HP1a). Input (Inp) is 10 and $5 \%$ of total cell extract. Asterisks indicate unspecific bands. (c) Direct interaction between His-SENP7 bound to beads and purified GST-HP1a.

Figure 2 SENP7 localizes with HP1a. (a) Endogenous SENP7 (green) and HP1a (red) localization in NIH3T3 cells extracted with Triton as indicated with $\mathrm{x} 3$ magnification of selected chromocenters (arrows). Scale bar, $10 \mu \mathrm{m}$. (b) Quantitative analysis of the percentage of cells with SENP7 and HP1a localized at pericentric domains or diffuse. Bars represent the mean and error bars indicate s.d. from 3 different experiments. (c) Left, SENP7 and HP1a detection in NIH3T3 cells treated with RNase A as indicated. Right, Western blot analysis of total proteins. (d) Left, SENP7 and HP1a detection in wild type and Suv39h double-null (dn) MEFs. Right, Western blot analysis of total proteins. Asterisks indicate unspecific bands. (e) Left, analysis of sumoylated HP1a-HA immunoprecipitate (GFP-SUMO1-HP1a-HA, black arrow and box), Asterisks indicate unspecific bands. Right, comparison of protein expression by Western blot. 
Figure 3 SENP7 depletion disrupts HP1 $\alpha$ localization at pericentric domains. (a) Experimental scheme. (b) Endogenous HP1a (blue) and SENP7 (red) localization in control (micont) and SENP7-depleted (miSENP7) GFP positive cells (green). Scale bar, $10 \mu \mathrm{m}$. (c) Quantitative analysis of the percentage of GFP positive cells with HP1a localized at pericentric domains or diffuse. Bars represent the mean and error bars indicate s.d. from 7 different experiments. $p_{\text {value }}=0.004$. (d) Endogenous HP1a (blue) and H3K9me3 (red) localization in GFP positive cells (green). (e) Quantitative analysis as in c. Bars represent the mean and error bars indicate s.d. from 3 different experiments. (f) Endogenous HP1 $\alpha$ (green) and SENP6 (red) localization in control (sicont) and siSENP6-treated (siSENP6) cells. (g) Quantitative analysis as in c. Bars represent the mean and error bars indicate s.d. from 3 different experiments.

\section{ONLINE METHODS}

Cells, miRNA and siRNA treatment, extracts. We cultured NIH3T3 cells and MEFs (provided by T. Jenuwein) ${ }^{13}$ in DMEM (Gibco BRL) containing $10 \%(\mathrm{v} / \mathrm{v})$ FCS at $37^{\circ} \mathrm{C}$ and $5 \% \mathrm{CO}_{2}$. We performed RNase treatment as described in ${ }^{12}$. We transfected NIH3T3 cells with Lipofectamin 2000 (Invitrogen) and performed analyses $72 \mathrm{~h}$ after transfection. We prepared total cell extract by resuspending cells in RIPA buffer (50 mM Tris- $\mathrm{HCl}$ pH 7.5, 150 $\mathrm{mM} \mathrm{NaCl}, 5 \mathrm{mM}$ EDTA, $15 \mathrm{mM} \mathrm{MgCl} 2,1 \%(\mathrm{v} / \mathrm{v})$ Nonidet P-40, and $0.75 \%(\mathrm{w} / \mathrm{v})$ sodium deoxycholate, supplemented with protease and phosphatase inhibitors, and $20 \mathrm{mM}$ NEM) and nuclear extracts as described $\mathrm{in}^{17}$.

Plasmids. To generate GST-SENP7 and His6-SENP7, we cloned mouse SENP7 cDNA obtained from I.M.A.G.E clone collection (isoform 1, clone ID IRAVp968H0599D6, RZPD) into pGEX-4T1 and pET-30a vector (Novagen). We made GFP-SENP7 by inserting SENP7 cDNA in the pEGFP-C3 vector (Invitrogen). The catalytic-mutant GFP-SENP7C979S was 
generated from GFP-SENP7 by site directed mutagenesis (Genescript). We used the pEGFP-C3 plasmid to express GFP controls. HP1a-HA expressing plasmid was as described in ${ }^{5}$. GFP-SUMO1, GFP-SUMO-2, GFP-SENP6 and GFP-SENP6C1030S ${ }^{18}$ were kindly provided by R. Hay. We constructed the plasmid expressing the miRNA targeting SENP7 (miSENP7) according to the Block-iT ${ }^{\mathrm{TM}}$ Pol II miR RNAi expression vector kit (Invitrogen) with the sequence 5'-TGATGAAGAAAGTTGCTCTGA-3'. Control miRNA (micont) with the sequence 5'-GAAATGTACTGCGCGTGGAGA-3' predicted not to target known vertebrate genes was provided with the Block-iT ${ }^{\mathrm{TM}}$ Pol II miR RNAi system (Invitrogen). The sequences of the siRNA were as follows: siSENP7, 5'ACAAGAAGCCUAAGAAAUA-3'; siSENP6, ON-TARGETplus SMARTpool J-062052-05, 06, 07 and 08 (Dharmacon); sicontrol, 5'-CGUACGCGGAAUACUUCGA-3'.

Immunofluorescence and image acquisition. We processed cells for immunostaining as described $^{17,19}$. For simultaneous visualisation of GFP with SENP7 and HP1a or H3K9me3 and HP1a, cells were not extracted prior to fixation. HP1a was detected with Alexa Fluor 647 secondary antibodies and SENP7 or H3K9me3 with Alexa Fluor 594 secondary antibodies. We used a Zeiss Z1 epifluorescence microscope equipped with a x63 objective lens and a chilled CCD camera (H2Q2, Ropper) for image acquisition.

Statistical analysis. The $p_{\text {value }}$ were calculated in $R$ program. We used the Student paired-t test to compare the \% of cells with HP1 localization at pericentric domains between miSENP7 and micont from 7 independent experiments. We counted more than 100 nuclei for each case per experiment. Differences were considered significant when $p_{\text {value }}<0.05$.

Antibodies and immunoprecipitation. We generated a rabbit polyclonal anti-mSENP7 by injecting recombinant full-length His6-mSENP7 (Agro-Bio). We tested its specificity for SENP7 by immunofluorescence and Western blot after depletion of SENP7 (Supplementary Fig. 2a,b). Rabbit serum was used at 1:1,000 dilution for Western blot. For 
immunofluorescence we affinity purified the antibodies by incubating $200 \mu$ l of serum with 5 $\mu \mathrm{g}$ of purified His6-mSENP7 present on a nitrocellulose membrane according to ${ }^{20}$, and used this purified antibody at 1:10 dilution. We used the following antibodies: for immufluorescence, mouse monoclonal anti-HP1a (2HP-1H5-AS, Euromedex; 1:1,000), rabbit polyclonal anti-H3K9me3 (\#07-442, Upstate; 1:1,000), goat polyclonal anti-SENP6 (\#ab77619, Abcam; 1:50) and secondary antibodies Alexa Fluor 568, 594 or 647 (Invitrogen); for Western blot, rabbit polyclonal anti-HP1a (C7F11, Cell signaling; 1:1,000), mouse monoclonal anti-HP1a (2HP-2G9-AS, Euromedex ; 1:1,000), mouse monoclonal antiHP1ß (1MOD-1A9-AS, Euromedex; 1:1,000), mouse monoclonal anti-HP1Y (2MOD-1G6, Euromedex; 1:1,000), goat polyclonal anti-SENP6 (\#ab77619, Abcam; 1:250), mouse monoclonal anti-GST (3G10/1B3, Abcam, 1/1,000), mouse monoclonal anti- $\beta$-actin (AC-15, Sigma; 1:20,000), rat monoclonal anti-HA (\#1867423, Roche; 1:2,000), mouse monoclonal anti-GFP (7.1 and $13.1 \mathrm{mix}$, Roche; 1:1,000), rabbit polyclonal anti-KAP1 (\#A300-274A, Bethyl; 1:1,000), rabbit polyclonal anti-SetDB1 (\#07-378, Upstate ; 1:1,000) and secondary antibodies coupled to horseradish peroxidase (Jackson Immuno Research Laboratories, Inc). We performed Western blot immunodetection on the membrane with the Super Signal kit (Pierce). We performed GFP and HP1a immunoprecipitation with anti-HA agaroseconjugated beads (Roche) and GFP-trap_A beads (Chromotek). We performed HA immunoprecipitations in RIPA buffer as in $^{5}$. We performed GFP precipitations and washes in $10 \mathrm{mM}$ Tris- $\mathrm{HCl} \mathrm{pH}$ 7.5, $500 \mathrm{mM} \mathrm{NaCl}, 0.5 \mathrm{mM}$ EDTA, $15 \mathrm{mM} \mathrm{MgCl}_{2}$ and $0.1 \%(\mathrm{v} / \mathrm{v})$ Nonidet P-40 supplemented with protease inhibitors. We quantified HP1a-HA and GFP-SUMO-1HP1a-HA from Western blots with a Chemi-doc XRS system and the QuantityOne software (Biorad).

Cell cycle/DNA content analysis by flow cytometry. After washes in PBS supplemented with $0.1 \%(w / v)$ BSA and $0.02 \%(w / v)$ sodium azide (PBA), we resuspended NIH3T3 cells with continuous gentle vortexing in $70 \%(\mathrm{v} / \mathrm{v})$ ethanol at $-20^{\circ} \mathrm{C}$ and incubated them on ice for $30 \mathrm{~min}$. Then we stained cells with 20 ug.ml-1 propidium iodide plus 10 ug.ml-1 RNase A in 
PBA for 30 min. We performed analysis on a FACSCalibur (Becton Dickinson) or on a C6 (Accuri) cytometer and FlowJo software (Treestar). We selected cells for analysis on their cycle profile by first gating on live cells with a forward scatter (FSC) vs side scatter (SSC) gate, then excluding multiplets with a SSC-H vs SSC-W gate and finally selecting either GFP-positive or GFP-negative cells.

Recombinant proteins and pull down. We bacterially expressed GST, GST-HP1 ${ }^{12}$, GSTSENP7, and His-SENP7 from E. coli BL21 (DE3) strain and immobilized proteins on glutathione (Amersham) or Ni-NTA beads (Novagen). We purified GST and GST-HP1a by elution from the glutathione beads according to manufacturer's instructions in buffer A (50 $\mathrm{mM}$ Tris- $\mathrm{HCl} \mathrm{pH} 7.5,250 \mathrm{mM} \mathrm{NaCl}, 0,05 \%(\mathrm{v} / \mathrm{v})$ Nonidet P-40, supplemented with protease inhibitors). We performed GST pull-down by mixing 3T3 nuclear extract with GST or GSTSENP7 beads and incubating for 2 hours at $4^{\circ} \mathrm{C}$ on a rotating wheel in buffer A. After five washes in buffer A containing $500 \mathrm{mM} \mathrm{NaCl}$, we recovered proteins by boiling in SDS-PAGE loading buffer and analyzed by Western-blot. We performed direct interaction assay by mixing GST or GST-HP1a with His-SENP7 beads and proceeded as above.

\section{REFERENCES}

1. Singh, P.B. et al. Nucleic Acids Res. 19, 789-94 (1991).

2. Kwon, S.H. \& Workman, J.L. Mol Cells 26, 217-27 (2008).

3. Nozawa, R.S. et al. Nat Cell Biol 12, 719-27 (2010).

4. $\quad$ Grewal, S.I. \& Jia, S. Nat Rev Genet 8, 35-46 (2007).

5. Maison, C. et al. Nat Genet 43, 220-7 (2011).

6. Kim, J.H. \& Baek, S.H. Biochim Biophys Acta 1792, 155-62 (2009).

7. Yeh, E.T. J Biol Chem 284, 8223-7 (2009).

8. Mukhopadhyay, D. \& Dasso, M. Trends Biochem Sci 32, 286-95 (2007).

9. Hay, R.T. Trends Cell Biol 17, 370-6 (2007).

10. Wilkinson, K.A. \& Henley, J.M. Biochem J 428, 133-45 (2010).

11. Shen, L.N., Geoffroy, M.C., Jaffray, E.G. \& Hay, R.T. Biochem J 421, 223-30 (2009).

12. Maison, C. et al. Nat Genet 30, 329-34 (2002).

13. Peters, A. et al. Cell 107, 323-337 (2001).

14. Prasanth, S.G., Shen, Z., Prasanth, K.V. \& Stillman, B. Proc Natl Acad Sci U S A 107, 15093-8 (2010).

15. Mikolajczyk, J. et al. J Biol Chem 282, 26217-24 (2007).

16. Lee, Y.K., Thomas, S.N., Yang, A.J. \& Ann, D.K. J Biol Chem 282, 1595-606 (2007).

17. Martini, E., Roche, D.M., Marheineke, K., Verreault, A. \& Almouzni, G. J Cell Biol 143, 563-75 (1998). 
18. Hattersley, N., Shen, L., Jaffray, E.G. \& Hay, R.T. Mol Biol Cell 22, 78-90 (2011).

19. Quivy, J.P. et al. EMBO J 23, 3516-26 (2004).

20. Smith, D.E. \& Fisher, P.A. J. Cell Biol. 99, 20-28 (1984). 

a

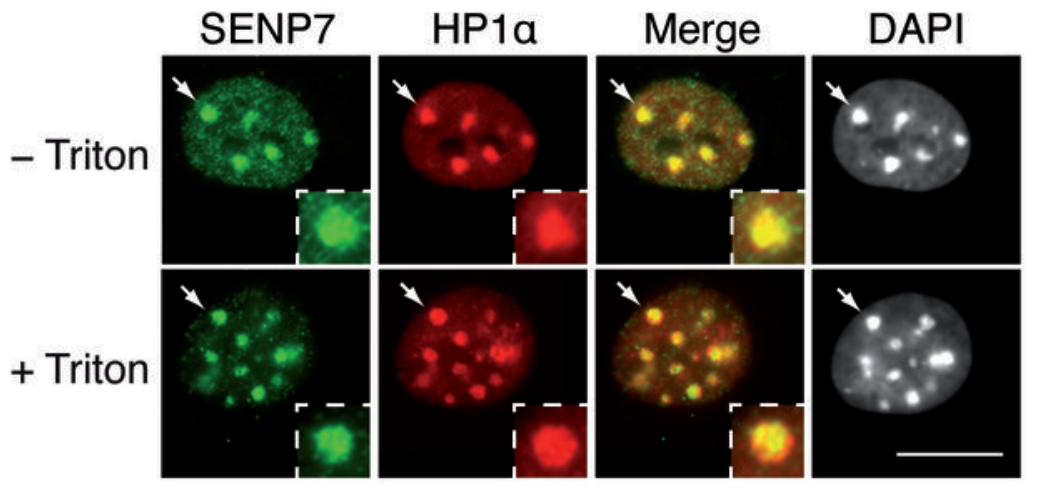

C

- RNase

+ RNase
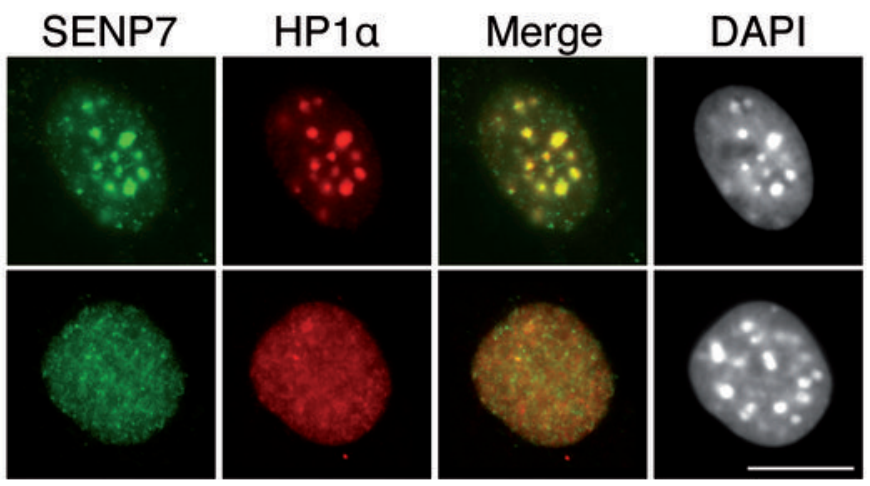

d
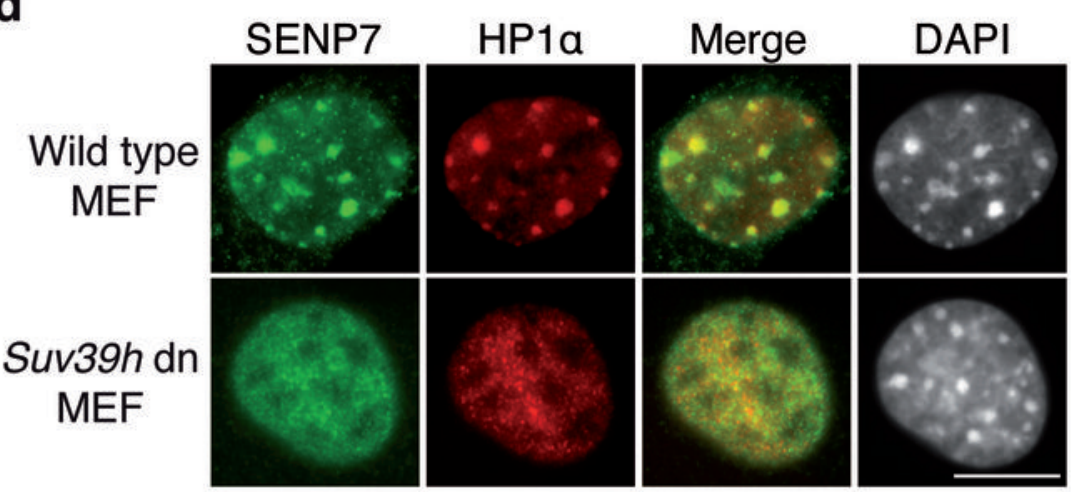
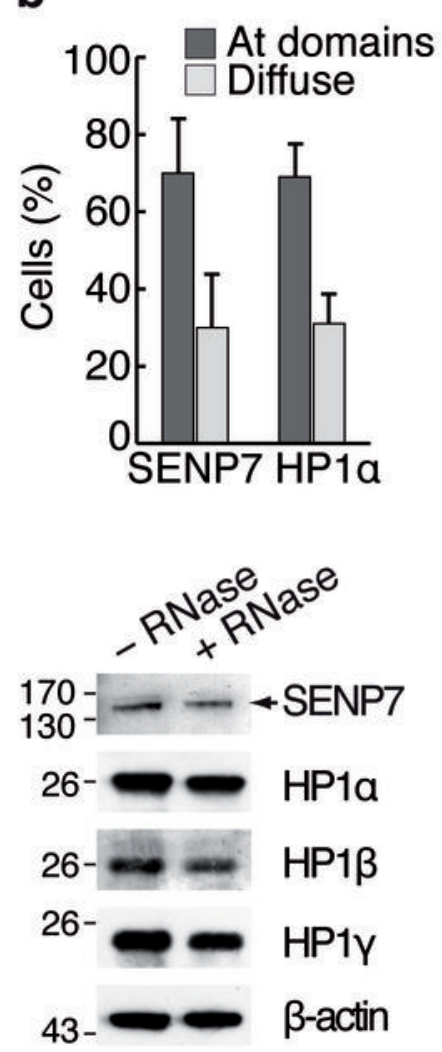

e

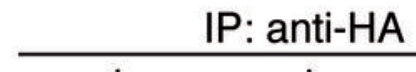

$\begin{array}{lllllllll}- & + & - & - & + & - & - & + & - \\ - & - & + & - & - & + & - & - & + \\ + & + & + & + & + & + & + & + & + \\ + & + & + & + & + & + & + & + & +\end{array}$

GFP-SENP7

GFP-SENP7C979S

GFP-SUMO-1

HP1a-HA
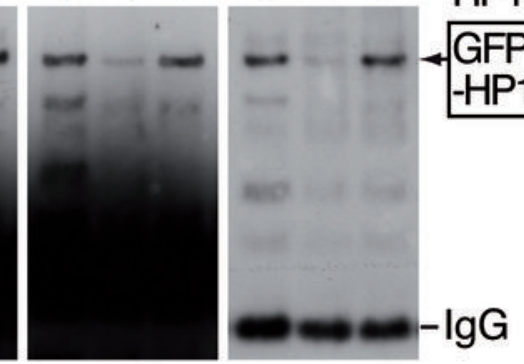

Anti-HA Anti-HP1a Anti-GFP
Total extract

$$
\begin{aligned}
& \overline{-}+\bar{c} \text { GFP-SENP7 } \\
& \overline{+}+\text { GFP-SENP7C979S } \\
& ++ \pm \text { GFP-SUMO-1 } \\
& ++ \pm \text { HP1a-HA }
\end{aligned}
$$

170- - 료 -GFP-SENP7 2 Endogenous SENP7]Anti-SENP7

$35-=-\mathrm{HP1a}-\mathrm{HA} \quad]$ Anti-HA

$26-2=-$ Endogenous HP1a $]$ Anti-HP1a

43-GFP-SUMO-1 ]Anti-GFP

43- $-\beta$ actin

]Anti- $\beta$ actin 
a $\quad \operatorname{miRNA} \mid \cdot \operatorname{micont}($ control)

Q GFP miRNA

b

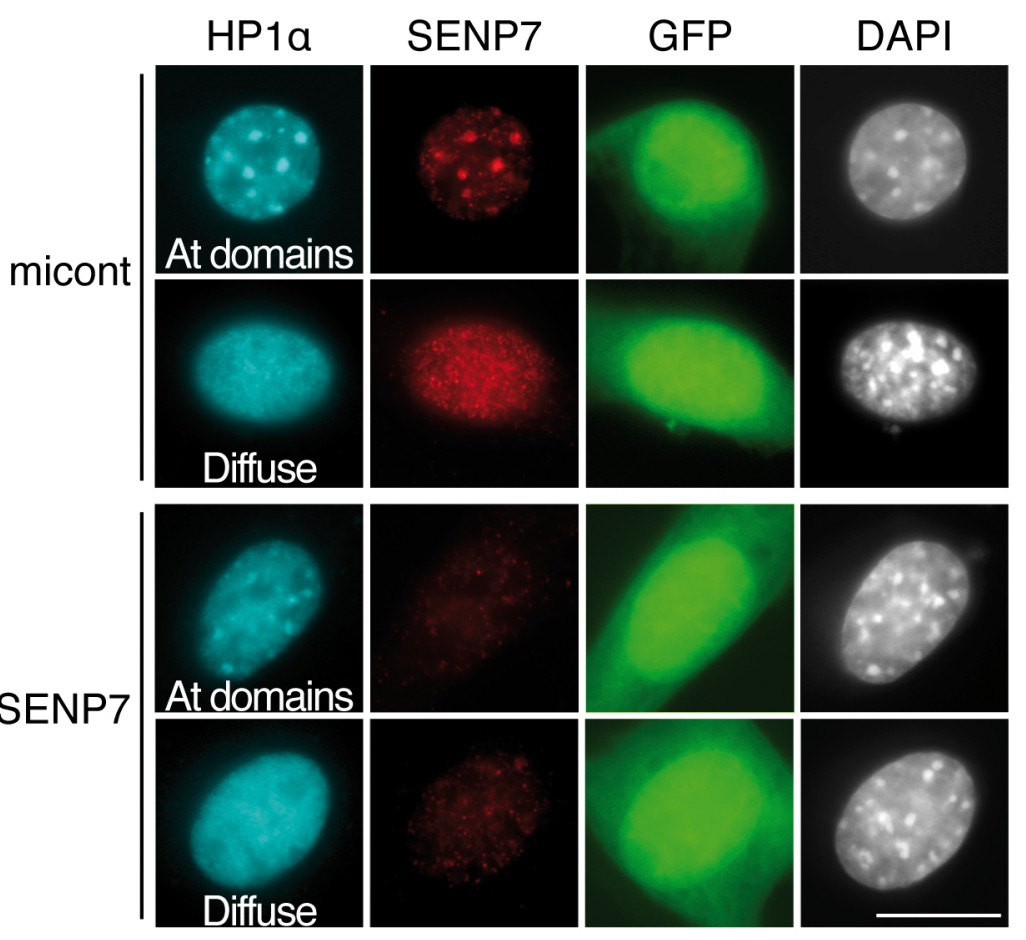

d

miSENP7
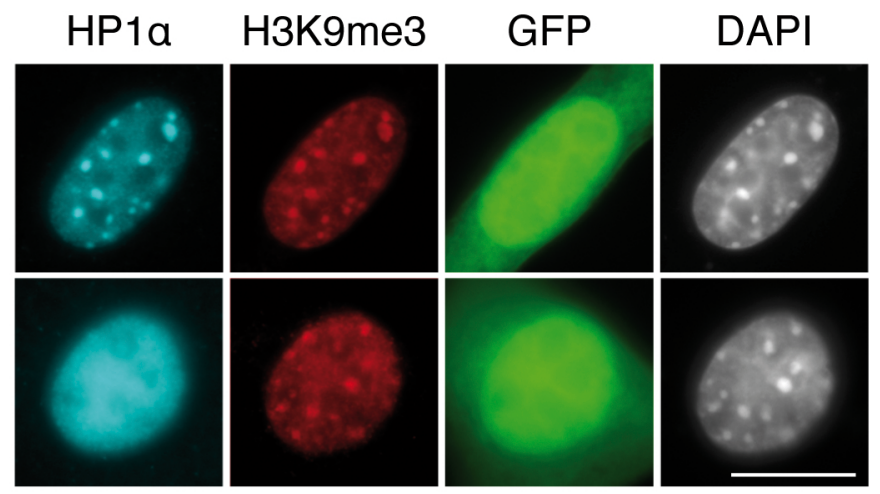

$\overbrace{}^{\circ} 100[\mathrm{HP} 1 \mathrm{a}\{\square$ Diffuse

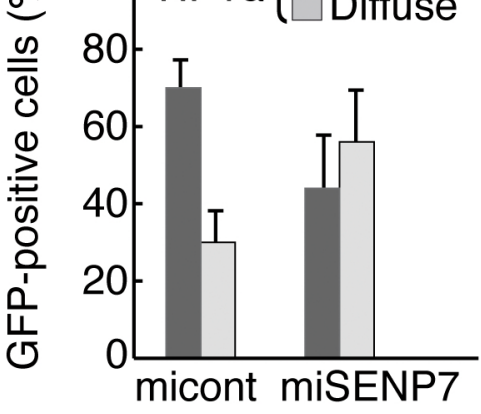

f

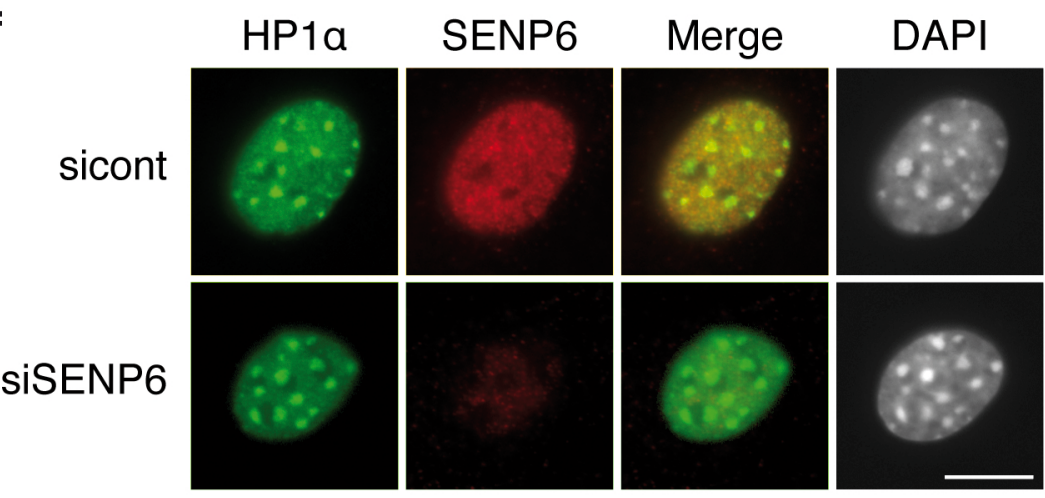

c

Control

SENP7

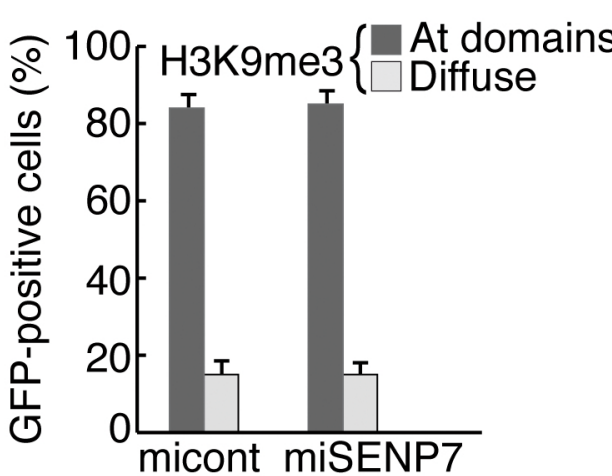

g

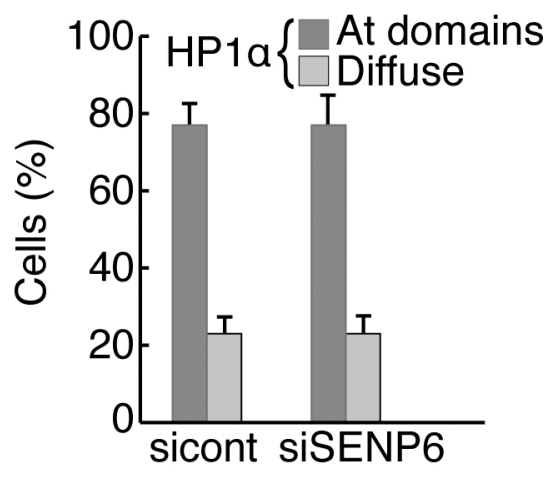

Maison Figure 3 


\section{SUPPLEMENTARY DATA}

The SUMO protease SENP7 is a critical component to ensure HP1 enrichment at pericentric heterochromatin

Christèle Maison*, Kelly Romeo*, Delphine Bailly, Marion Dubarry, Jean-Pierre Quivy ${ }^{\ddagger}$ and Geneviève Almouzni ${ }^{\ddagger}$ 
a

IP: anti-GFP
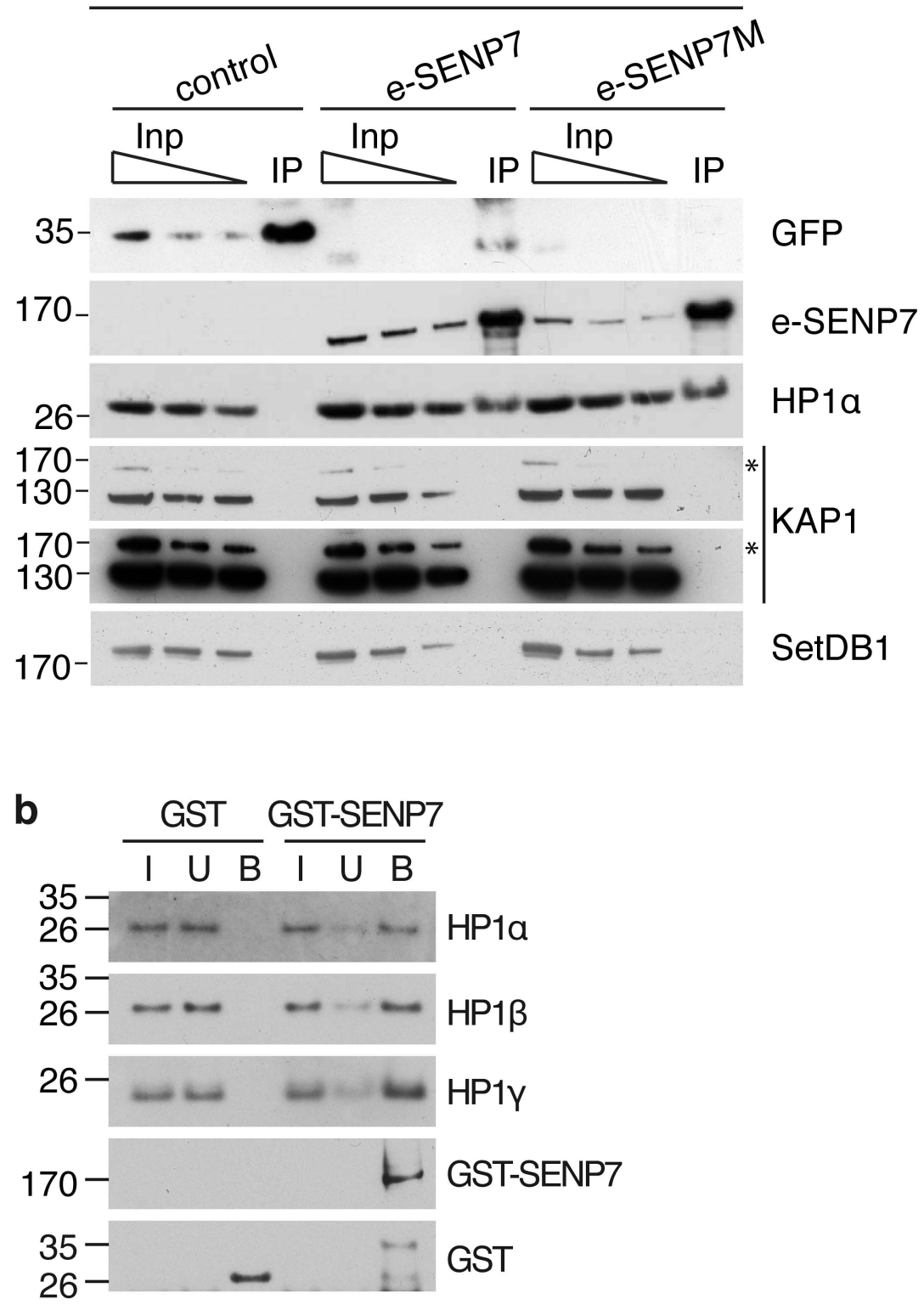

Supplementary Figure 1 SENP7 interacts with HP1. (a) Immunoprecipitation (IP) of GFP (control), GFP-SENP7 wild-type (e-SENP7) and GFP-SENP7C979S (e-SENP7M). We performed Western blot using anti-GFP, anti-HP1a, anti-KAP1 and anti-SetDB1 antibodies to reveal proteins in the input (Inp) and the immunoprecipitates (IP). We show 2 exposures for KAP1 detection. Input (Inp) is 5, 2.5, 1.25\% of total cell extract. Asterisk indicates unspecific band. (b) We performed GST and GST-SENP7 pulldown from NIH3T3 nuclear extract. Western blot revealed HP1a, HP1 $1 \beta$, HP1Y, GST and GST-SENP7 in the input $(\mathrm{I}, 10 \%)$, unbound $(\mathrm{U})$ and bound $(\mathrm{B})$ fractions. 
NIH3T3 cells

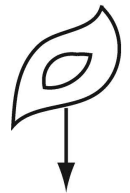

Co-transfection

HP1a-HA

$+$

- GFP or GFP-SUMO-1

- +/- GFP-SENP7

$72 \mathrm{~h} \downarrow$

Total extract (+ NEM)

IP: anti-HA

Western

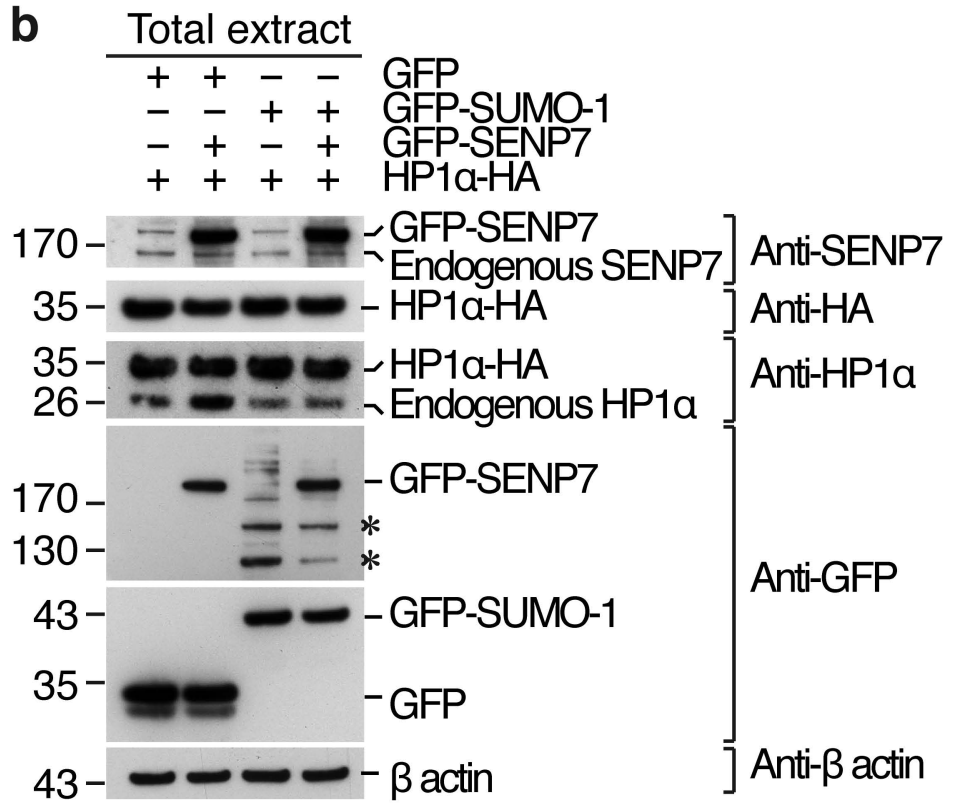

C

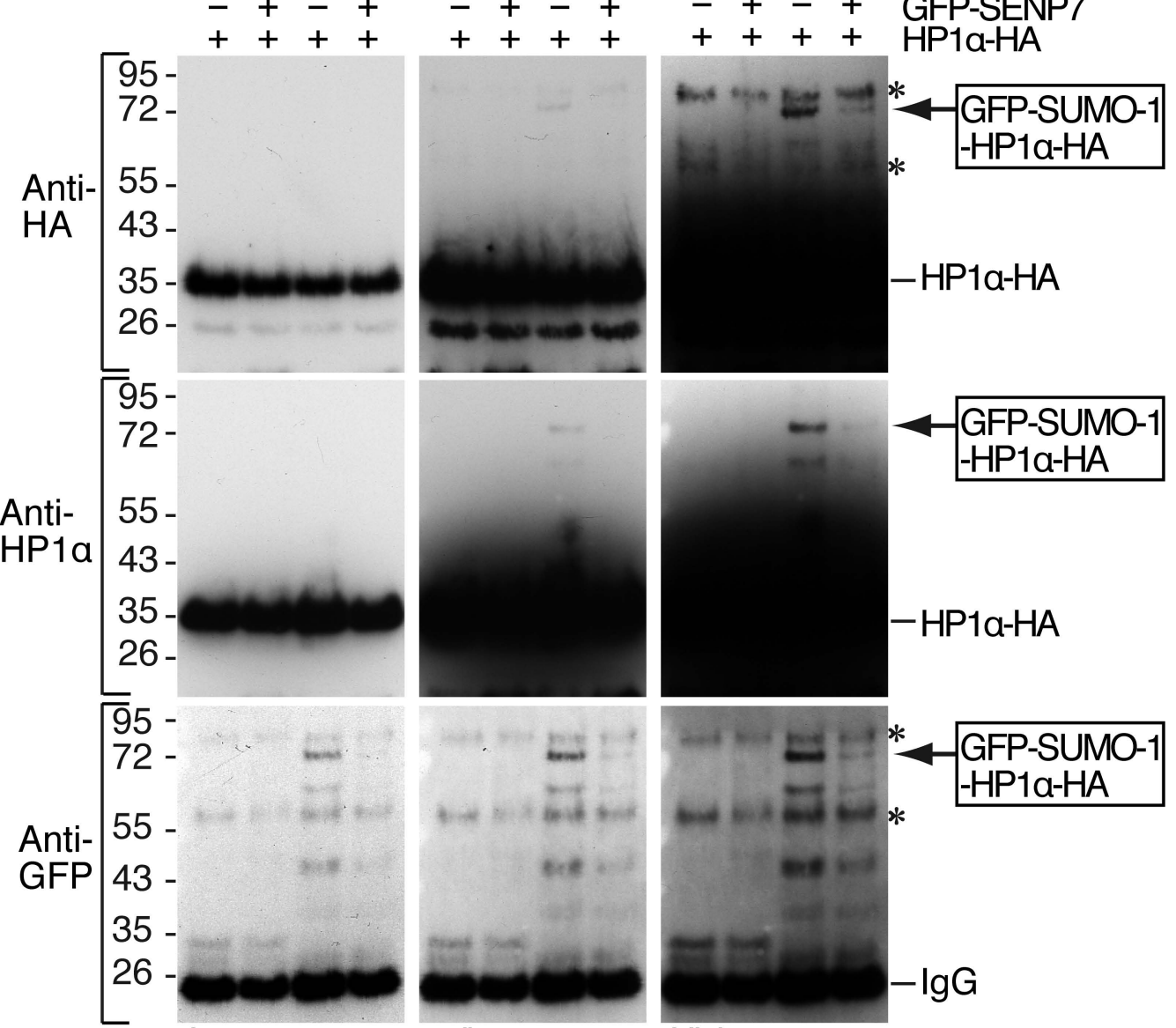

Low exposure medium exposure High exposure d

Ratio

$\frac{\text { GFP-SUMO-1-HP1a-HA }}{\text { HP1a-HA }}$
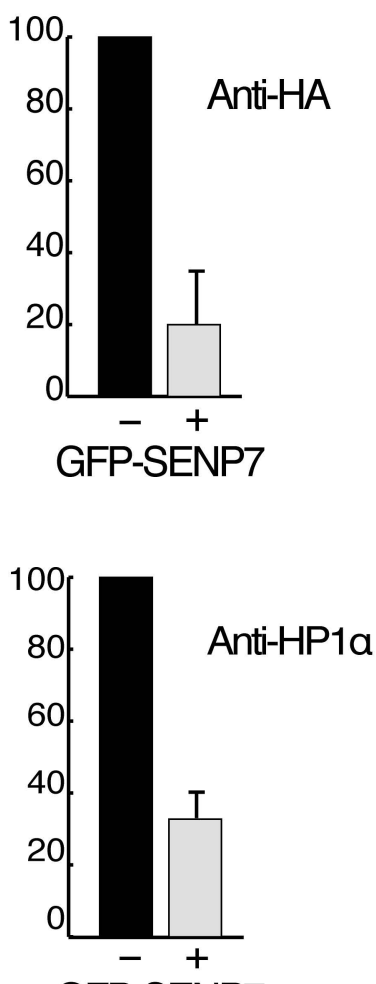

GFP-SENP7

Supplementary Figure 2 SENP7 deconjugates SUMO-1-HP1a in vivo. (a) Experimental scheme. (b) We transfected NIH3T3cells with GFP, GFP-SUMO-1, GFP-SENP7, HP1a-HA as indicated. We verified the expression of the proteins in total cell extracts using anti-SENP7, anti-HA, anti-HP1a, anti-GFP and anti- $\beta$-actin antibodies. Asterisks indicate unspecific bands. (c) After anti-HA immunoprecipitation, Western blot using anti-HA, anti-HP1 $a$ and anti-GFP antibodies revealed sumoylated HP1a-HA (GFP-SUMO-1-HP1 a-HA, black arrow and box) and unmodified HP1a-HA. We show low, medium and high exposures for each detection. IgG corresponds to the immunoglobulin light chain. Asterisks indicate unspecific bands. (d) Quantification of the decrease of GFP-SUMO1-HP1a-HA levels. An histogram showing comparison of the ratio between GFP-SUMO-1-HP1a-HA and unmodified HP1a-HA in the absence (-) or presence (+) of GFP-SENP7 is represented. We performed quantifications with anti-HA (top) or anti-HP1a (bottom) antibodies. The $100 \%$ is set for the control (absence of GFP-SENP7). The bars represent the mean and error bars indicate the s.d. from 5 and 2 different experiments for anti-HA and anti-HP1a detections respectively. 
a micont miSENP7

SENP7
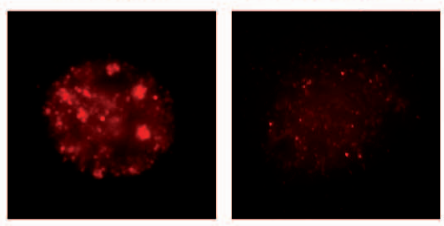

DAPI
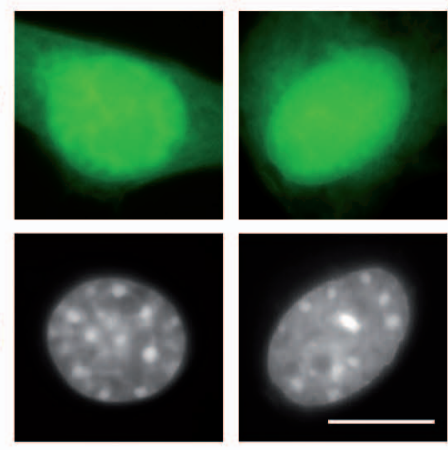

d

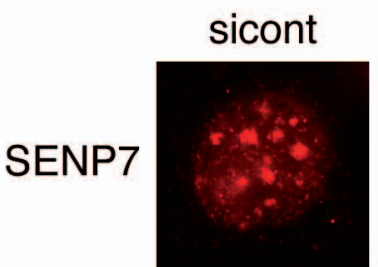

siSENP7

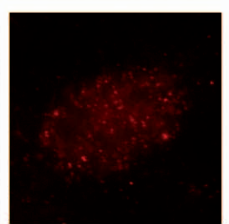

HP1a
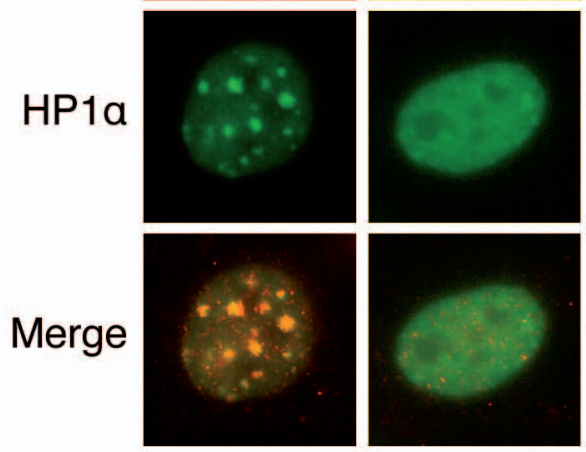

DAPI
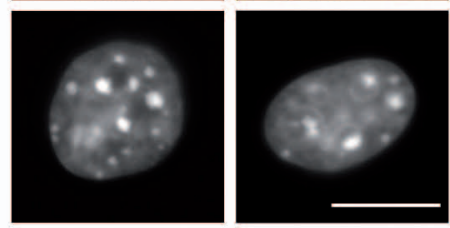
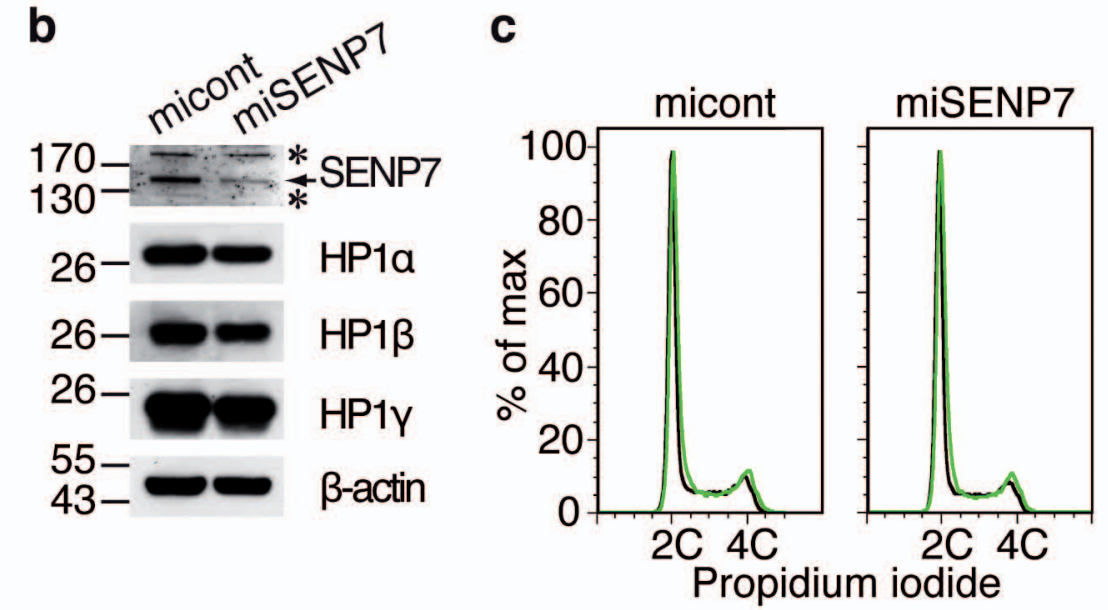

GFP-positive a GFP-negative

e
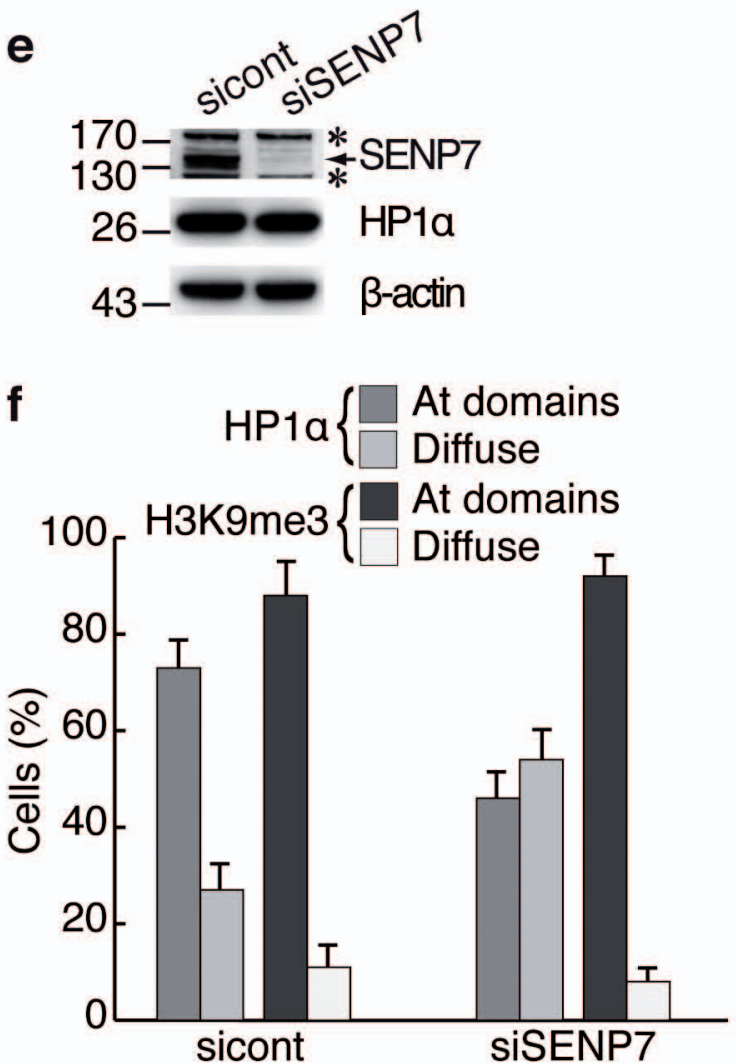

g

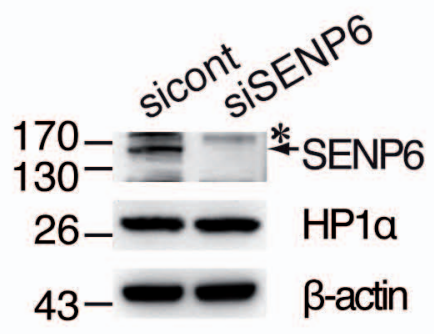

Supplementary Figure 4 SENP7 downregulation in NIH3T3 cells. (a) Endogenous SENP7 (red) localization in control (micont) and SENP7-depleted (miSENP7) GFP positive cells (green). Scale bar, 10 $\mu \mathrm{m}$. (b) Western blot of total proteins from cells transfected as in a. Since we did not select the GFPpositive cells, the presence of SENP7 (arrow) in the miSENP7 extract arises from non-transfected cells. Asterisks indicate unspecific bands. (c) Flow cytometry analysis of the DNA content of NIH3T3 cells transfected as in a. (d) Endogenous SENP7 (red) and HP1a (green) localization in control (sicont) and siSENP7-treated (siSENP7) cells. Scale bar, $10 \mu \mathrm{m}$. (e) Western blot of total proteins from cells transfected as in d. Asterisks indicate unspecific bands. (f) Quantitative analysis of the percentage of cells with HP1 $\mathrm{a}$ and $\mathrm{H} 3 \mathrm{~K} 9 \mathrm{me} 3$ localized at pericentric domains or diffuse from 3 different experiments. (g) Western blot of total proteins from control (sicont) and SENP6-depleted (siSENP6) cells. Asterisk indicates unspecific band. 
a NIH3T3 cells

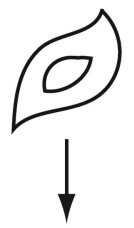

Co-transfection

HP1a-HA
+
- GFP or GFP-SUMO-1
- +- GFP-SENP6 or C1030S

$72 \mathrm{~h}$

Total extract (+ NEM)

IP: anti-HA

Western b Total extract

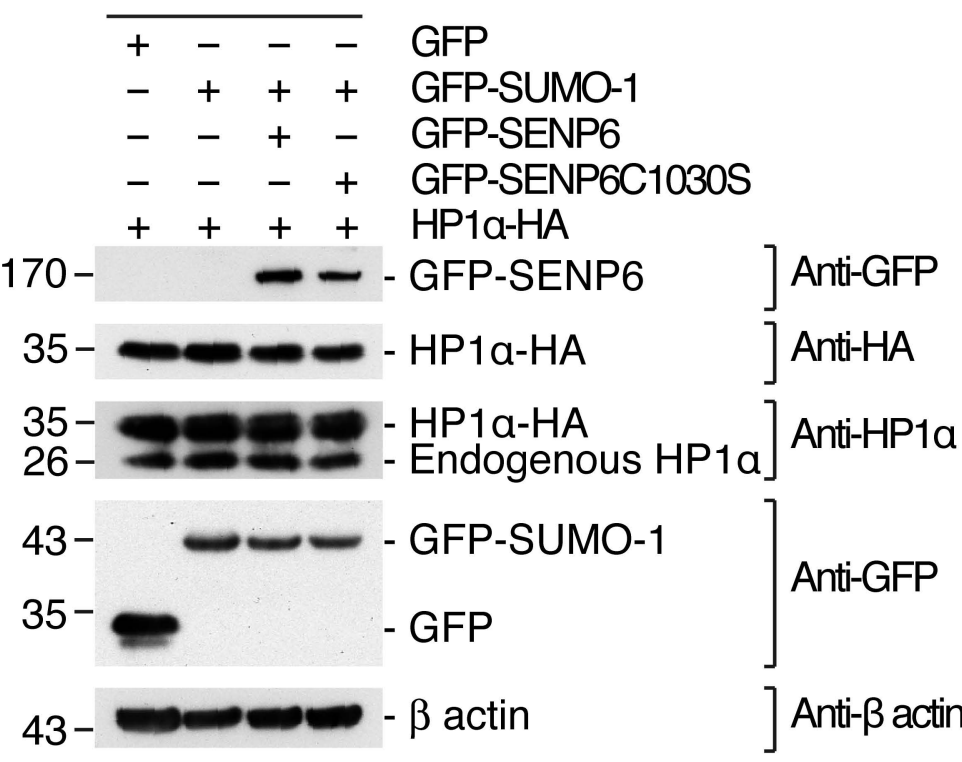

-+++ GFP-SUMO-1

- ++ - GFP-SENP6

-+-+ GFP-SENP6C1030S

HP1a-HA

GFP-SENP6

] Anti-GFP

] Anti-HA

Anti-HP1a

Anti-GFP

Anti- $\beta$ actin

IP: anti HA

72 -
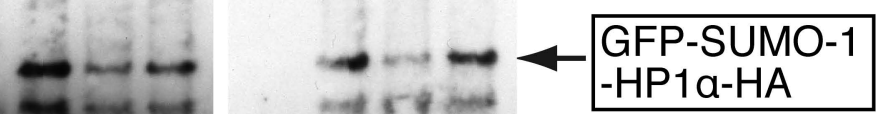

55 -

43.

35 -

\begin{tabular}{lllllllllllll}
\hline+ & - & - & - & + & - & - & - & + & - & - & - & GFP \\
- & + & + & + & - & + & + & + & - & + & + & + & GFP-SUMO-1 \\
- & - & + & - & - & - & + & - & - & - & + & - & GFP-SENP6 \\
- & - & - & + & - & - & - & + & - & - & - & + & GFP-SENP6C1030S \\
+ & + & + & + & + & + & + & + & + & + & + & + & HP1a-HA
\end{tabular}

$\ldots \cdots$

Supplementary Figure 5 SENP6 deconjugates SUMO-1-HP1a in vivo. (a) Experimental scheme. (b) We transfected NIH3T3cells with GFP, GFP-SUMO-1, GFP-SENP6, GFPSENP6C1030S and HP1a-HA as indicated. We verified the expression of the proteins in total cell extracts using anti-HA, anti-HP1a, anti-GFP and anti- $\beta$-actin antibodies. (c) After anti-HA immunoprecipitation, Western blot using anti-GFP, anti-HA and anti-HP1 $a$ antibodies revealed sumoylated HP1a-HA (GFP-SUMO-1-HP1a-HA, black arrow and box) and unmodified HP1a-HA. 
b Total extract

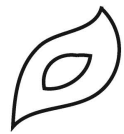

+--- GFP

-+++ GFP-SUMO-2

$-\quad+-$ GFP-SENP7

$\downarrow$

$-\quad-\quad+$ GFP-SENP7C979S

++++ HPla-HA

Co-transfection

HP1a-HA
+
- GFP or GFP-SUMO-2
$\cdot+/-$ GFP-SENP7 or C979S

$72 \mathrm{~h} \downarrow$

Total extract (+ NEM)

IP: anti-HA

Western

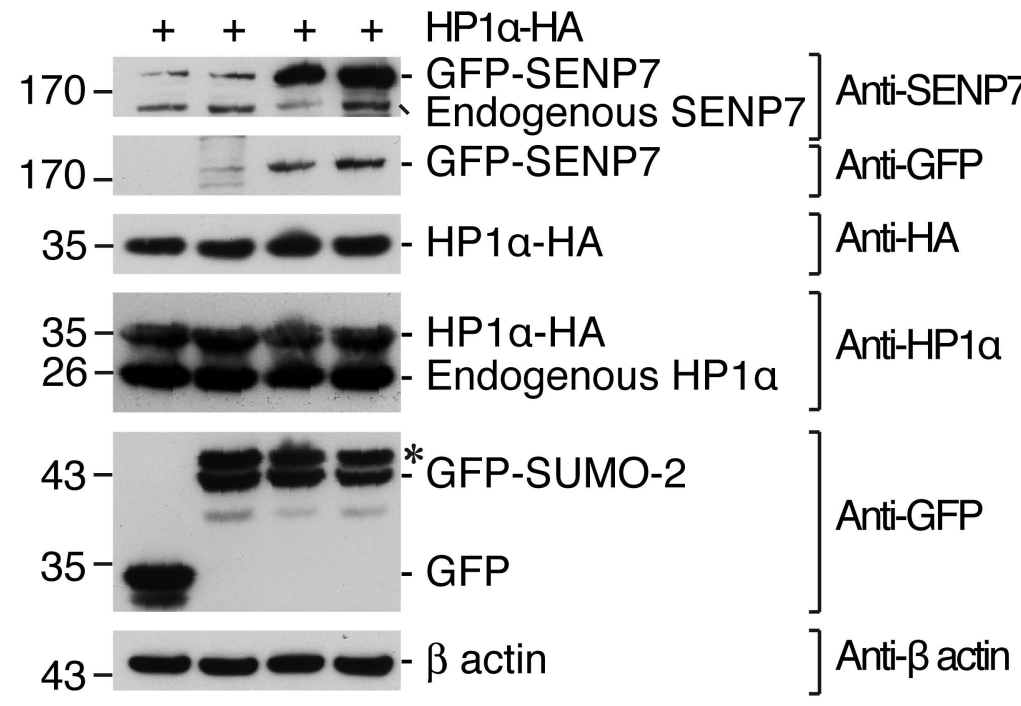

IP: anti HA

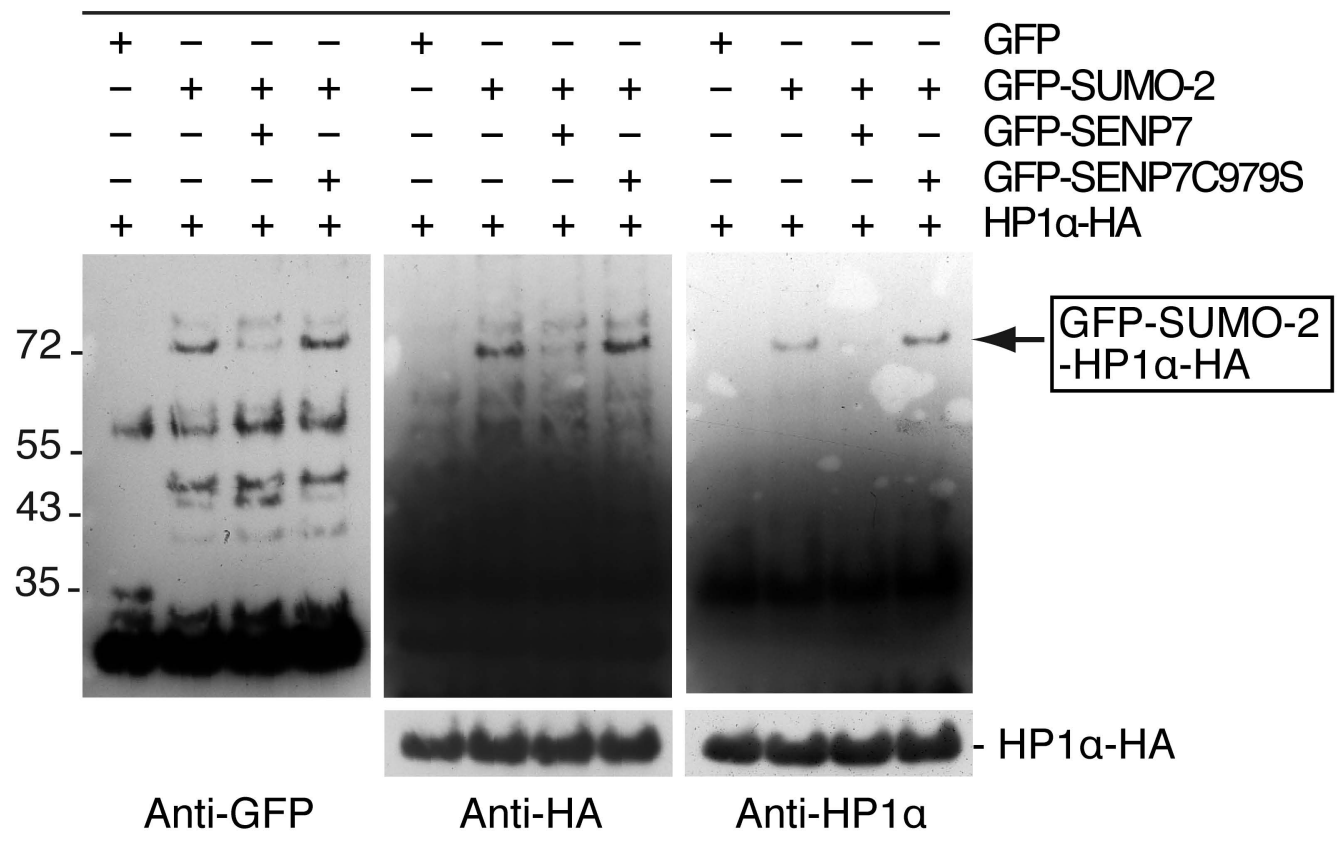

Supplementary Figure 6 SENP7 deconjugates SUMO-2-HP1a in vivo. (a) Experimental scheme. (b) We transfected NIH3T3cells with GFP, GFP-SUMO-2, GFP-SENP7, GFPSENP7C979S and HP1a-HA as indicated. We verified the expression of the proteins in total cell extracts using anti-SENP7, anti-HA, anti-HP1a, anti-GFP and anti- $\beta$-actin antibodies. Asterisk indicates unspecific band. (c) After anti-HA immunoprecipitation, Western blot using anti-GFP, anti-HA and anti-HP1a antibodies revealed sumoylated HP1a-HA (GFP-SUMO-2-HP1a-HA, black arrow and box) and unmodified HP1a-HA. 